\title{
CESÀRO SUMMABILITY OF FOURIER SERIES
}

\author{
KôSI KANNO \\ (Received October 10,1954)
}

Let $\varphi(t)$ be an even periodic function with Fourier series

$$
\varphi(t) \sim \sum_{n=0}^{\infty} a_{n} \cos n t, a_{0}=0 .
$$

The $\alpha$-th integral of $\phi(t)$ is defined by

$$
\varphi_{\alpha}(t)=\frac{1}{\Gamma(\alpha)} \int_{0}^{t} \varphi(u)(t-u)^{\alpha-1} d u \quad(\alpha>0) .
$$

G. Sunouchi [1] has proved the following theorem ${ }^{1)}$;

Theorem 1. Let $\Delta=\gamma / \beta \geqq 1$. If

$$
\varphi_{3}(t)=o\left(t^{\gamma}\right) \quad(t \rightarrow 0)
$$

and further if

$$
\int_{0}^{t}\left|d\left(u^{د} \varphi(u)\right)\right|=O(t), \quad 0<t<\eta,
$$

then the Fourier series of $\phi(t)$ converges to zero at $t=0$.

Concerning this theorem M. Kinukawa [4] has proved the following theorem;

THEOREM 2. Let $\Delta \geqq 1,-1<\alpha<1$. If

$$
\begin{gathered}
\gamma=\Delta-\frac{2 \alpha(\Delta-1)}{1+\alpha}, \\
\int_{0}^{t} \phi(u) d u=o\left(t^{\prime}\right)
\end{gathered}
$$

and

$$
\int_{0}^{t}\left|d\left(u^{د} \varphi(u)\right)\right|=O(t), \quad 0<t<\eta
$$

then the Fourier series of $\varphi(t)$ is summable $(C, \alpha)$ to zero at $t=0$.

The object of this paper is to generalize the above theorems.

THEOREM ${ }^{2)}$. If

$$
\left.\phi_{3}(t)=o_{(}^{\prime} t^{\gamma}\right), \quad \gamma>\beta>0,
$$

and

1) An Alternative proof was given by Prof. S. Izumi [5].

2) This theorem was proposed by Prof. G. Sunouchi. 


$$
\int_{0}^{t}\left|d\left(u^{د} \phi(u)\right)\right|=O(t), \quad 0<t<\eta
$$

then the Fourier series of $\phi(t)$ is summable $(C, \alpha)$ to zero at $t=0$, where $\alpha=\frac{\Delta \beta-\gamma}{\Delta+\gamma-\beta-1}$ and $\Delta \geqq \frac{\gamma}{\beta}$.

If we put $\Delta=\frac{\gamma}{\beta}$, we have Theorem 1 . And if we put $\beta=1$, then $\alpha=\frac{\Delta-\gamma}{\Delta+\gamma-2}$, that is, $\gamma=\Delta-\frac{2 \alpha(\Delta-1)}{1+\alpha}$. This is Theorem 2 in the case $0<\alpha<1$.

Proof. We use Bessel summability instead of Cesàro summability: Accordingly, the proof is due to Sunouchi's method [2].

Let $J_{\mu}(t)$ denote the Bessel function of order $\mu$, and put

$$
\begin{aligned}
\alpha_{\mu}(t) & =J_{\mu} / t^{\mu} \\
V_{1+\mu}(t) & =\alpha_{\mu+1 / 2}(t)
\end{aligned}
$$

then

$$
\begin{gathered}
V_{1+\mu}^{(k)}(t)=O(1) \quad \text { as } t \rightarrow 0 \quad \text { and } \\
V_{1+\mu}^{(k)}(t)=O\left(t^{-(\mu+1)}\right) \quad \text { as } t \rightarrow \infty, \text { for } k=0,1,2, \ldots \ldots
\end{gathered}
$$

We denote by $\sigma_{\omega}^{\alpha}$ the $\alpha$-th Bessel mean of the Fourier series (1). Since the case $\alpha=0$ is Theorem 1 , we may suppose that $\alpha>0$. Neglecting the constant factor,

$$
\sigma_{\omega}^{\alpha}=\int_{0}^{\infty} \omega \varphi(t) V_{1+\alpha}(\omega t) d t=\left(\int_{0}^{C_{\omega}-\rho}+\int_{\omega_{\omega}-\rho}^{\infty}\right) \omega \varphi(t) V_{1+\alpha}(\omega t) d t=I+J,
$$

say, where $C$ is a fixed large constant and $\rho=\frac{\alpha+1}{\alpha+\Delta}<1$.

If we put

$$
\theta(t)=t^{د} \phi(t), \quad \Theta(t)=\int_{0}^{t}|d \theta(t)|
$$

then we have, by (4)

$$
\Theta(t)=O(t), \quad \theta(t)=O(t)
$$

Next we consider the formula [6]

$$
\int_{0}^{\infty} \frac{J_{\nu}\left(a \sqrt{t^{2}+z^{2}}\right)}{\left(t^{2}+z^{2}\right)^{\nu / 2}} t^{2 \mu+1} d t=2^{\mu} \Gamma(\mu+1) \frac{J_{\nu-\mu+1}(a z)}{a^{\nu+1} z^{\nu-\mu+1}},
$$

where $a>0$, 註 $\left(\frac{\nu}{2}-\frac{1}{4}\right)>\Re(\mu)>-1$.

In the above formula, if we put $t^{2}+z^{2}=\tau^{2}, \mu=\lambda$, then

$$
\int_{z}^{\infty} \frac{J_{\nu}(a \tau)\left(\tau^{2}-z^{2}\right)^{\lambda}}{\tau^{\nu-1}} d \tau=2^{\lambda} \Gamma(\lambda+1) J_{\nu-\lambda-1}(a z) /\left(a^{\lambda+1} z^{\nu-\lambda-1}\right)
$$


where $a>0, \mathfrak{H}\left(\frac{\nu}{2}-\frac{1}{4}\right) \quad \mathfrak{R}(\lambda)>-1$.

This formula is valid when $\lambda=0, \nu>1 / 2$. Therefore

$$
\int_{z}^{\infty} \frac{J_{\nu}(a \tau)}{\tau^{\nu-1}} d \tau=J_{\nu-1}(a z) / a z^{\nu-1}
$$

Now, by (8) and (9)

$$
\begin{aligned}
\Lambda(t)=\int_{t}^{\infty} \frac{V_{1+\alpha}(\omega t)}{u^{\Delta}} d u & =\int_{t}^{\infty} \frac{J_{\alpha+1 / 2}(\omega u)}{u^{\Delta}(\omega u)^{\alpha+1 / 2}} d u \\
& =\omega^{-(\alpha+1 / 2)} \int_{t}^{\infty} \frac{J_{\alpha+1 / 2}(\omega u)}{u_{\alpha-1 / 2} u^{\Delta+1}} d u .
\end{aligned}
$$

By (10) and (13), integrating by parts we get

$$
\begin{aligned}
\omega^{(\alpha+1 / 2)} \Lambda(t)= & {\left[-\int_{u}^{\infty} \frac{J_{\alpha+1 / 2}(\omega v)}{v^{\alpha-1 / 2}} d v \cdot u^{-(\Delta+1)}\right]_{t}^{\infty} } \\
& -(\Delta+1) \int_{t}^{\infty}\left\{\int_{u}^{\infty} \frac{J_{\alpha+1 / 2}(\omega v)}{v^{\alpha-1 / 2}} d v\right\} u^{-(\Delta+2)} d u \\
= & {\left[J_{\alpha-1 / 2}(\omega u) \omega^{-1} u^{-(\alpha-1 / 2)} u^{-(\Delta+1))}\right]_{t}^{\infty} } \\
& \quad-(\Delta+1) \omega^{-1} \int_{t}^{\infty} J_{\alpha-1 / 2}(\omega u) u^{-(\alpha-1 / 2)} u^{-(\Delta+2)} d u \\
= & O\left\{\left[\omega^{-1}(\omega u)^{-1 / 2} u^{-(\alpha+\Delta+1 / 2)}\right]_{t}^{\infty}\right\}+O\left\{\int_{t}^{\infty} \omega^{-3 / 2} u^{-1 / 2} u^{-(\Delta+\alpha+3 / 2)} d u\right\} \\
= & O\left(\omega^{-3 / 2} t^{-(\Delta+\alpha+1))}+\left(O \omega^{-3 / 2} t^{-(\Delta+\alpha+1)}\right),\right.
\end{aligned}
$$

for $\omega t>1$. Thus if $\omega t>1$, then we have

(14)

$$
\Lambda(t)=O\left(\omega^{-(\alpha+2)} t^{-(\Delta+\alpha+1)}\right) .
$$

We first estimate $J$. By integration by parts, we have

$$
\begin{aligned}
J & =\int_{C_{\omega}-\rho}^{\infty} \omega \varphi(t) V_{1+\alpha}(\omega t) d t=\int_{C_{\omega}-\rho}^{\infty} \omega \theta(t) \frac{V_{1+\alpha}(\omega t)}{t^{\Delta}} d t \\
& =-\int_{c_{\omega}-\rho}^{\infty} \omega \theta(t) d \Lambda(t)=-[\theta(t) \omega \Lambda(t)]_{C_{\omega}-\rho}^{\infty}+\omega \int_{\omega_{\omega}-\rho}^{\infty} \Lambda(t) d \theta(t) \\
& =J_{1}+J_{2},
\end{aligned}
$$

say. Then, by (12) and (14)

$$
\begin{aligned}
J_{1} & =O\left[\omega t \omega^{-(\alpha+2)} t^{-(\Delta+\alpha+1)}\right]_{\omega^{-\rho}}^{\infty}=O\left(\omega^{-(\alpha+1)} C^{-(\Delta+\alpha)} \omega^{\rho(\Delta+\alpha)}\right) \\
& =O\left(C^{-(\Delta+\alpha)}\right) \leqq \varepsilon
\end{aligned}
$$


for large $C$, since $\rho=\frac{1+\alpha}{\Delta+\alpha}$.

$$
\begin{aligned}
& J_{2}=O\left\{\int_{C \omega}^{\infty} \omega^{-(\alpha+1)} t^{-(\Delta+\alpha+1)}|d \theta(t)|\right\} \\
& =O\left\{\omega^{-(\alpha+1)}\left[\Theta(t) t^{-(\Delta+\alpha+1)}\right]_{C_{\omega}-\rho}^{\infty}+\int_{C_{\omega}-\rho}^{\infty} \omega^{-(\alpha+1)} \Theta(t) t^{-(\Delta+\alpha+2)} d t\right\} \\
& \left.=O\left\{\omega^{-(\alpha+1)}\left(C \omega^{-\rho}\right)^{-(\Delta+\alpha)}+\omega^{-(\alpha+1)}\left[t^{-(\Delta+\alpha)}\right]_{C \omega}^{\infty}\right\}^{\infty}\right\} \\
& =O\left\{\omega^{-(\alpha+1)} C^{-(\Delta+\alpha)} \omega^{\rho(\Delta+\alpha)}\right\} \\
& =O\left(C^{-(\Delta+\alpha)}\right) \leqq \varepsilon \text {. }
\end{aligned}
$$

Thus

$$
J=J_{1}+J_{2} \leqq \varepsilon .
$$

Now there is an integer $k>1$ such that $k-1<\beta \leqq k$. We suppose that $k-1<\beta<k$, for the case $\beta=k$ can be easily deduced by the following argument. By integration by parts $k$-times, we have

$$
\begin{aligned}
I & =\sum_{h=1}^{k}(-1)^{h-1}\left[\omega^{h} \varphi_{h}(t) V_{1+\alpha}^{(h-1)}(\omega t)\right]_{0}^{c_{\omega}^{-\rho}}+(-1)^{k} \omega^{k+1} \int_{0}^{c_{\omega}-\rho} \boldsymbol{\phi}_{k}(t) V_{1+\alpha}^{(k)}(\omega t) d t \\
& =\sum_{h=1}^{k}(-1)^{h-1} I_{h}+(-1)^{k} I_{k+1}, \text { say. }
\end{aligned}
$$

Since $\varphi(t)=O\left(t^{1-\Delta}\right)$ by (12) and $\phi_{\beta}(t)=o\left(t^{\gamma}\right)$, we have, by convexity theorem due to G. Sunouchi [3],

$$
\phi_{h}(t)=o\left(t^{((\beta-h)(1-\Delta)+h \gamma) / \beta}\right), \text { for } h=1,2, \ldots k-1,
$$

Therefore, if $\beta>1$

$$
\varphi_{k}(t)=o\left(t^{\gamma-\beta+k}\right) .
$$

$$
\begin{aligned}
& \boldsymbol{I}_{h}=\left[\boldsymbol{\omega}^{h} \phi_{h}(t) V_{1+\alpha}^{(h-1)}(\omega t)\right]_{0}^{c_{\omega}-\rho} \\
& =o\left\{\omega^{h-(1+\alpha)} \omega^{-\kappa((\beta-h)(1-\Delta)+h \gamma\} / \beta} \omega^{\rho(1+\alpha)} C^{((\beta-h)(1-\Delta)+h \gamma\} / \beta} C^{-(1+\alpha)\}}\right\} \\
& +\omega^{h} \lim _{t \rightarrow 0} V_{1+\alpha}^{h-1}(\boldsymbol{\omega} t) t^{((\beta-h)(1-\Delta)+h \gamma) / \beta} .
\end{aligned}
$$

Now, if the condition (7) holds then the Fourier series of $\varphi(t)$ is summable $\left(C, \frac{\beta}{1+\gamma-\beta}\right)$ to zero at $t=0$. Therefore if

$\frac{\beta}{1+\gamma-\beta}>\frac{\Delta \beta-\gamma}{\Delta+\gamma-\beta-1}$, that is $\frac{\gamma+\beta+1}{\beta}>\Delta$, then our theorem has the meaning. Hence we may suppose $\frac{\gamma+\beta+1}{\beta}>\Delta$.

If $\beta>1$ we have $\frac{\gamma+\beta-1}{\beta-1}>\frac{\gamma+\beta+1}{\beta}$. Thus we have $(\beta-1)(1-\Delta)$ $+\gamma>0$. 
Since $(\beta-h)(1-\Delta)+h \gamma>(\beta-1)(1-\Delta)+\gamma>0$ and $V_{1+\alpha}^{(h-1)}(\omega t)=O(1)$ as $t \rightarrow 0$ the second term is zero. Since $\rho=(1+\alpha) /(\Delta+\alpha)=(\beta+1) /(\Delta+\gamma)$ the $\omega$ 's exponent of the first term is

$$
\begin{aligned}
& h-(1+\alpha)-\frac{\rho}{\beta}\{(\beta-h)(1-\Delta)+h \gamma-\beta(1+\alpha)\} \\
&= h-(1+\alpha)-\frac{\rho}{\beta}\{-\beta(\alpha+\Delta)-h(1-\Delta-\gamma)\} \\
&= h-(1+\alpha)+\frac{\rho}{\beta} \frac{\beta(1+\alpha)}{\rho}-\frac{\rho}{\beta} h(\Delta+\gamma-1) \\
&= \frac{h}{\beta}\left\{\beta-\frac{(\beta+1)(\Delta+\gamma-1)}{\Delta+\gamma}\right\}=\frac{h}{\beta(\Delta+\gamma)}(\beta+1-\Delta-\gamma)<0, \\
&(h=1,2,3, \ldots k-1) . \quad \text { If } \beta<1 \\
& I_{1}= {\left[\omega \varphi_{1}(t) V_{1+\alpha(\omega t)}\right]_{0}^{(\omega-\rho}=O\left\{\omega^{1-(1+\alpha)} \omega^{-\rho((\beta-1)(1-\Delta)+\gamma\} / \beta} \omega^{\rho(1+\alpha)}\right\} } \\
& \quad \cdot C^{((\beta-1)(1-\Delta)+\gamma\} / \beta} C^{-(1+\alpha)}-\lim _{t \rightarrow 0} \omega t^{\{(\beta-1)(1-\Delta)+\gamma\} / \beta} V_{1}^{+\alpha}(\omega t) .
\end{aligned}
$$

Since $(\beta-1)(1-\Delta)+\gamma>0$ and $V_{1+\alpha}(\omega t)=O(1)$ as $t \rightarrow 0$, the second term is zero. About the $\omega$ 's expont of the first term we have

$$
(\beta+1-\Delta-\gamma) / \beta(\Delta+\gamma)<0
$$

by similar calculation. In this case another terms of $I_{h}$ disappear for $h=$ $2,3, \ldots k-1$. Thus we have

$$
I_{h}=o(1), \text { as } \omega \rightarrow \infty \quad \text { for } h=1,2, \ldots k-1 .
$$

Concerning $I_{k}$,

$$
\begin{aligned}
\boldsymbol{I}_{l k} & =\left[\omega^{k} \varphi_{k}(t) V_{1+\alpha}^{(k-1)}(\omega t)\right]_{0}^{c \omega^{-\rho}} \\
& =o\left\{\omega^{k} \omega^{-\rho(k+\gamma-\beta)} \omega^{-(1+\alpha)} \omega^{\rho(1+\alpha)}\right\}-\lim _{t \rightarrow 0} \omega^{k} t^{(k+\gamma-\beta)} V_{1+\alpha}^{(k-1)}(\omega t) \\
& =o\left\{\omega^{k(1-\rho)-\rho(\gamma-\beta)-(1-\rho)(1+\alpha)}\right\}
\end{aligned}
$$

The exponent of $\omega$ is

$$
\begin{aligned}
& \frac{k(\Delta-1)}{\Delta+\alpha}-\frac{1+\alpha}{\Delta+\alpha}(\gamma-\beta)-\frac{\Delta-1}{\Delta+\alpha}(1+\alpha) \\
= & \frac{k(\Delta-1)}{\Delta+\alpha}-\frac{1+\alpha}{\Delta+\alpha}(\gamma+\Delta-\beta-1) \\
= & \frac{\Delta-1}{\Delta+\alpha}(k-\beta-1)=(1-\rho)(k-\beta-1)<0,
\end{aligned}
$$

for $1+\alpha=(\Delta-1)(\beta+1) /(\gamma+\Delta-\beta-1)$. Therefore

$$
I_{k}=o(1), \quad \text { as } \omega \rightarrow \infty .
$$

Concerning $I_{k+1}$, we split it up into four parts,

$$
I_{k+1}=\omega^{k+1} \int_{0}^{C_{\omega}-\rho} \varphi_{k}(t) V_{1+\alpha}^{(k)}(\omega t) d t=\omega^{k+1} \int_{0}^{C_{\omega}-\rho} V_{1+\alpha}^{(k)}(\omega t) d t
$$


CESÀRO SUMMABILITY OF FOURIER SERIES

115

$$
\begin{gathered}
\cdot \int_{0}^{t} \varphi_{\beta}(u)(t-u)^{k-\beta-1} d u \\
=\int_{0}^{C_{\omega}-\rho} \omega^{k+1} \varphi_{\beta}(u) d u \int_{u}^{C_{\omega}-\rho} V_{1+\alpha}^{(k)}(\omega t)(t-u)^{k-\beta-1} d t \\
=\int_{0}^{\omega^{-1}} d u \int_{u}^{u+\omega^{-1}} d t+\int_{\omega-1}^{C_{\omega}-\rho} d u \int_{u}^{u+\omega^{-1}} d t+\int_{0}^{C_{\omega}-\rho}-\omega^{-1} d u \int_{u+\omega^{-1}}^{C_{\omega}-\rho} d t-\int_{0}^{C_{\omega}-\rho} d u \int_{\omega_{\omega}-\rho}^{u+\omega^{-1}} d t \\
=K_{1}+K_{2}+K_{3}-K_{1},
\end{gathered}
$$

say. Since $V_{1+\alpha}^{(k)}(t)=O(1)$ for $0 \leqq t \leqq 1$,

$$
\begin{aligned}
K_{1} & =\omega^{k+1} \int_{0}^{\omega^{-1}} \phi_{\beta}(u) d u \int_{u}^{u+\omega^{-1}} V_{1+\infty}^{(k)}(\omega t)(t-u)^{k-\beta-1} d t \\
& =O\left\{\omega^{k+1} \int_{0}^{\omega^{-1}} \varphi_{\beta}(u) d u \int_{u}^{u+\omega^{-1}}(t-u)^{k-\beta-1} d t\right\} \\
& =o\left\{\omega^{k+1} \int_{0}^{\omega^{-1}} u^{\gamma}\left[(t-u)^{k-\beta}\right]_{u}^{u+\omega^{-1}} d u\right\} \\
& =o\left\{\omega^{k+1} \int_{0}^{\omega^{-1}} u^{\nu} \omega^{-(k-\beta)} d u\right\}=o\left\{\omega^{\beta+1}\left[u^{\gamma+1}\right]_{0}^{\omega^{-1}}\right\} \\
& =o\left(\omega^{\beta-\gamma}\right)=o(1), \text { for } \gamma>\beta .
\end{aligned}
$$

(19)

$$
\begin{aligned}
K_{2} & =\omega^{k+1} \int_{\omega^{-1}}^{C_{\omega}-\rho} \phi_{\beta}(u) d u \int_{u}^{u+\omega^{-1}} V_{1+\alpha}^{(k)}(\omega t)(t-u)^{k-\beta-1} d t \\
& =o\left\{\omega^{k+1} \int_{\omega^{-1}}^{C_{\omega}-\rho} u^{\gamma} d u \int_{u}^{u+\omega^{-1}}(\omega t)^{-(1+\alpha)}(t-u)^{k-\beta-1} d t\right\} \\
& =o\left\{\omega^{k-\alpha} \int_{\omega^{-1}}^{C_{\omega}-\rho} u^{\gamma} u^{-(1+\alpha)} \int_{u}^{u+\omega^{-1}}(t-u)^{k-\beta-1} d t\right\} \\
& =o\left\{\omega^{k-\alpha} \int_{\omega^{-1}}^{C_{\omega}-\rho} u^{\gamma-(1+\alpha)} d u\left[(t-u)^{k-\beta}\right]_{u}^{u+\omega^{-1}}\right\} \\
& =o\left\{\omega^{k-\alpha} \omega^{-(k-\beta)}\left[u^{\gamma-\alpha}\right]_{\omega^{-1}}^{C_{\omega}-\rho}\right\}=o\left(\omega^{\beta-\alpha} \omega^{-\rho(\gamma-\alpha)}\right),
\end{aligned}
$$

for $\gamma-\alpha=\gamma-\frac{\Delta \beta-\gamma}{\Delta+\gamma-\beta-1}=\frac{(\gamma-\beta)(\Delta+\gamma)}{\Delta+\gamma-\beta-1}>0$.

Since

$$
\beta-\alpha-\rho(\gamma-\alpha)=\frac{1}{\alpha+\Delta}\{\beta \Delta-\gamma-\alpha(\Delta+\gamma-\beta-1)\}=0,
$$

we have 
(20)

$$
K_{2}=o(1) \quad \text { as } \omega \rightarrow \infty .
$$

Concerning $K_{3}$, if we use integration by parts in the inner integral, then

$$
\begin{aligned}
K_{3}= & \omega^{k+1} \int_{c}^{\gamma_{\omega-\rho}-\omega^{-1}} \phi_{\beta}(u) d u \int_{u+\omega^{-1}}^{C \omega-\rho} V_{1+\alpha}^{(k)}(\omega t)(t-u)^{k-\beta-1} d t \\
= & \omega^{k+1} \int_{0}^{C \omega-\rho-\omega^{-1}} \phi_{\beta}(u) d u\left\{\left[\omega^{-1} V_{1+\alpha}^{(k-1)}(\omega t)(t-u)^{k-\beta-1}\right]_{u+\omega^{-1}}^{C \omega-\rho}\right. \\
& \left.-(k-\beta-1) \int_{u+\omega^{-1}}^{C \omega-\rho} V_{1+\alpha}^{(k-1)}(\omega t) \cdot(t-u)^{k-\beta-2} d t\right\} \\
= & M_{1}-(k-\beta-1) M_{2},
\end{aligned}
$$

say. Then

$$
\begin{gathered}
M_{1}=\omega^{k+1} \int_{0}^{C_{\omega}-\rho-\omega^{-1}} \varphi_{\beta}(u) d u\left\{\omega^{-1} \omega^{-(1+\alpha)} \omega^{\rho(1+\alpha)}\left(C \omega^{-\rho}-u\right)^{k-\beta-1}\right. \\
\left.-\omega^{-1} \omega^{-(1+\alpha)}\left(u+\omega^{-1}\right)^{-(1+\alpha)} \omega^{-(k-\beta-1)}\right\}
\end{gathered}
$$

(22) $=N_{1}+N_{2}$.

$$
\begin{aligned}
& N_{1}=o\left\{\omega^{k+(\rho-1)(1+\alpha)} \int_{0}^{C_{\omega}-\rho} u^{\gamma}\left(C \omega^{-\rho}-u\right)^{c-\beta-1} d u\right\} \\
& =o\left\{\omega^{k+(\rho-1)(1+\alpha)}\left[u^{\gamma+k-\beta}\right]_{0}^{c_{\omega}-\rho}\right\}=o\left(\omega^{k+(\rho-1)(1+\alpha)-\rho(\gamma+k-\beta)}\right) .
\end{aligned}
$$

Since the exponent of $\omega$ is

$$
\begin{aligned}
k & -\frac{(\Delta-1)(\alpha+1)}{\Delta+\alpha}-\frac{\alpha+1}{\Delta+\alpha}(\gamma+k-\beta) \\
& =\frac{1}{\Delta+\alpha}\{k(\Delta-1)-\alpha(\gamma+\Delta-\beta-1)-\gamma-\Delta+\beta+1\} \\
& =\frac{1}{\Delta+\alpha}\{k(\Delta-1)-(\beta \Delta-\gamma)-\gamma-\Delta+\beta+1\} \\
& =\frac{\Delta-1}{\Delta+\alpha}(k-\beta-1)=(1-\rho)(k-\beta-1)<0,
\end{aligned}
$$

$$
\begin{aligned}
& N_{1}=o(1) \quad \text { as } \omega \rightarrow \infty \\
& N_{2}=o\left\{\omega^{k-(1+\alpha)-(k-\beta-1)} \int_{0}^{C \omega^{-\rho}-\omega^{-1}} u^{\gamma}\left(u+\omega^{-1}\right)^{-(1+\alpha)} d u\right\} \\
&=o\left\{\omega^{\beta-\alpha} \int_{0}^{C \omega^{-\rho}} u^{\gamma-(1+\alpha)} d u\right\} \\
&=o\left(\omega^{3-\alpha} \omega^{-(\gamma-\alpha) \rho}\right)=o^{\prime}(1) \quad \text { as } \omega \rightarrow \infty .
\end{aligned}
$$

From (23) and (24) we have
(26)
$M_{1}=o(1)$
as $\omega \rightarrow \infty$ 


$$
\begin{aligned}
M_{2} & =\omega^{k} \int_{0}^{C_{\omega}-\rho-\omega^{-1}} \phi_{\beta}(u) d u \int_{u+\omega^{-1}}^{C_{\omega}-\rho} V_{1+\alpha}^{(k-1)}(\omega t)(t-u)^{k-\beta-2} d t \\
& =o\left\{\omega^{k} \int_{0}^{C_{\omega}-\rho}-\omega^{-1} u^{\gamma} d u \int_{u+\omega^{-1}}^{C_{\omega}-\rho} \omega^{-(1+\alpha)} t^{-(1+\alpha)}(t-u)^{k-\beta-2} d t\right\} \\
& =o\left\{\omega^{k-(1+\alpha)} \int_{0}^{C_{\omega}-\rho-\omega^{-1}} u^{\gamma} u^{-(1+\alpha)} d u \int_{u+\omega^{-1}}^{C_{\omega}-\rho}(t-u)^{k-\beta-2} d t\right\} \\
& =o\left\{\omega^{k-(1+\alpha)} \int_{0}^{C_{\omega}-\rho-\omega^{-1}} u^{\gamma-(1+\alpha)}\left[(t-u)^{k-\beta-1}\right]_{u+\omega^{-1}}^{C_{\omega}-\rho} d u\right\} \\
& =o\left\{\omega^{k-(1+\alpha)} \int_{0}^{C_{\omega}-\rho} u^{\gamma-(1+\alpha)} \omega^{-(k-\beta-1)} d u\right\} \\
& =o\left\{\omega^{k-(1+\alpha)-(k-\beta-1)}\left[u^{\gamma-\alpha}\right]_{0}^{C_{\omega}-\rho}\right\} \\
& =o\left(\omega^{\beta-\alpha} \omega^{-\rho(\gamma-\alpha)}\right)=o(1) \text { as } \omega \rightarrow \infty .
\end{aligned}
$$

From (21), (26) and (27) we have

(28)

$$
\begin{aligned}
& K_{3}=\propto(1) \quad \text { as } \omega \rightarrow \infty . \\
& K_{4}=\omega^{k+1} \int_{C_{\omega}-\rho_{-\omega}-1}^{C_{\omega}-\rho} \phi_{\beta}(u) d u \int_{C_{\omega}-\rho}^{u+\omega^{-1}} V_{1+\alpha}^{(k)}(\omega t)(t-u)^{k-\beta-1} d t \\
& =O\left\{\omega^{k+1} \int_{\omega_{\omega}-\rho_{-\omega}-1}^{C_{\omega}-\rho} \varphi_{\beta}(u) d u \int_{C_{\omega}-\rho}^{u+\omega^{-1}}(\omega t)^{-(1+\alpha)}(t-u)^{k-\beta-1} d t\right\} \\
& =O\left\{\omega^{k+1-(1+\alpha)} \int_{\omega_{\omega}^{-\rho}-\omega^{-1}}^{C_{\omega}-\rho} \phi_{\beta}(u) \omega^{\rho(1+\alpha)} d u \int_{\omega^{\omega} \omega^{-\rho}}^{u+\omega^{-1}}(t-u)^{k-\beta-1} d t\right\} \\
& =o\left\{\omega^{k-\alpha+\rho(1+\alpha)} \int_{C_{\omega}-\rho-\omega^{-1}}^{C_{\omega}-\rho} u^{\gamma}\left[(t-u)^{k-\beta}\right]_{C_{\omega}-\rho}^{u+\omega^{-1}} d u\right\} \\
& =o\left\{\omega^{k-\alpha+\rho(1+\alpha)} \omega^{-(k-\beta)}\left[u^{\gamma+1}\right]_{C \omega-\rho_{-\omega}^{-1}}^{C_{\omega}-\rho}\right\} \\
& =o\left(\omega^{k-\alpha+\rho(1+\alpha)-(k-\beta)} \omega^{-\rho(\gamma+1)}\right)=o\left(\omega^{\beta-\alpha-\rho(\gamma-\alpha)}\right) .
\end{aligned}
$$

Since the exponent of $\omega$ is

$$
\begin{aligned}
& \beta-\alpha-\rho(\gamma-\alpha) \\
& =\frac{1}{\Delta+\alpha}\{\beta(\Delta+\alpha)-\alpha(\Delta+\alpha)-(\alpha+1)(\gamma-\alpha)\} \\
& =\frac{1}{\Delta+\alpha}\{\beta \Delta-\gamma-\alpha(\gamma+\Delta-\beta-1)\}=0,
\end{aligned}
$$

(29) $\quad K_{4}=o(1) \quad$ as $\omega \rightarrow \infty$.

Summing up (19), (20), (28) and (29) we have 
(30)

$$
I_{k+1}=o(1)
$$

as $\omega \rightarrow \infty$

From (11), (15), (17), (18) and (30) we have

$$
\sigma_{\omega}^{\alpha}=\alpha(1) \quad \text { as } \omega \rightarrow \infty
$$

which is required.

\section{REFERENCES}

[1] G. SunouchI, A new convergence criterion for Fourier series, Tôhoku Math. Journ., 5(1954).

[2] G. SUNOUCHI, Cesàro summability of Fourier series, Tôhoku Math. Journ., 5(1953).

[3] G. SUNOUCH, Convexity theorems and Cesàro summability of Fourier series, Journ. of Math., 1(1953).

[4] M. KInUKAWA, On the Cesàro summability of Fourier series, Tôhoku Math. Journ., 6(1954).

[5] S. IZUMI, Some trigonometrical series VIII, Tôhoku Math. Journ., 5(1954).

[6] G. N. WATSON, Theory of Bessel functions, 2nd edition, Cambridge, 1944.

DEPARTMENT OF MATHEMatics, FacUlty of Liberal ARTS AND Science, YAMAGATA UNJVERSITY 